\title{
To Investigate the Extent of the Level of Physical Activity Participation and Lifestyle Choice having a Significant Influence on Body Image Perception and Awareness in University Students Aged 18 - 21
}

\section{Lewis Bridges* and Dr Stephen Pack}

School of Life and Medical Sciences, University of Hertfordshire, England, United

Kingdom

*Corresponding Author: Lewis Bridges MSc BSc (Hons).
Received: April 29, 2021

Published: May 20, 2021

(C) All rights are reserved by Lewis Bridges and Dr Stephen Pack.

\begin{abstract}
Introduction: Body image refers to the perception of physical appearance and the external factors which may create low body confidence. The aim of this study was to investigate the extent of the level of physical activity participation and lifestyle choice having an influence on body image perception and awareness in University students aged 18 - 21, which is believed to dictate one's variation in behaviour and attitude.

Methods: Forty participants were recruited through random opportunistic sampling and were placed into Competitive Active (University team), Social Active (non-competitive), Casual Active (exercise at home/gym) and Sedentary test groups. The Body Awareness Questionnaire, Body Consciousness Questionnaire and Social Physique Anxiety Scale were given on one occasion as part of a descriptive cross-sectional study.

Results: Trends were expected with consistent findings supporting the inclusion age, showing body image presentation can be indicative of an initial external judgement. Social and Casual groups do not have the outcome achievement goal (Competitive) but are self-motivated to better performance, or to socialise/compete with friends. There were no statistically significant differences between group means for each questionnaire ( $\mathrm{p}>0.05)$.

Conclusion: The level of physical activity and lifestyle choice does have a moderate influence on body image perception and awareness, in both independent and social scenarios. Sedentary people create a negative impression of themselves over time and psychologically adjust to their physical appearance.
\end{abstract}

Keywords: Body Image; Perception; Awareness; Physical Activity; Lifestyle; Confidence; Independence; Social; Anxiety

\section{Research Study Aims and Hypotheses}

The aim of this study was to investigate the extent of a relationship between the level of physical exercise undertaken during a typical week and one's lifestyle choice on body image perception and awareness in University students aged 18 - 21 (late adolescence). This will consider the psychological aspects behind the attitudes and behaviors towards body presentation and conscious emotion relative to physical appearance, in both personal/ conscious states and social scenarios, through quantitative data collection. 
The experimental hypotheses are:

1. The people who participate in competitive active will be more satisfied with body awareness, compared with social active: The body awareness questionnaire (BAQ) will be used, which is an 18-item scale, with the total scale score calculated as the sum of the items, with each individual item scored on a 1 - 7 scale, with questions being reverse scored, meaning if one marked a score of 1 , it would be processed as a score of 5 . Higher scores dictate high attentiveness and awareness to normal body processes and emotions.

2. There will be a difference in body consciousness between social active and casual active due to increased self-drive for personal outcomes: The body consciousness questionnaire (BCQ) will be used, which involves three sub-scales, public body consciousness (6 questions), private body consciousness ( 5 items) and body competence (5 items). They are responded to on a 5-point Likert scale, ranging from 0 (extremely uncharacteristic) to 4 (extremely characteristic). Higher scores represent greater body awareness/competence.

3. Sedentary individuals have a reduced concern for their body awareness due to developing a negative impression of themselves, compared with the physically active: (The BCQ will be used, with further details explained above).

4. Competitive active people will be less influenced by social scenarios compared with casually active people: The Social Physique Anxiety Scale (SPAS) will be used, which is a 12 -item self-report scale developed to assess the degree to which people become anxious when others observe or evaluate their physiques (physical appearance). It is scored from 1 (low) to 5 (high). Higher scores dictate greater anxiety during real evaluation of one's physique.

The null hypothesis $\left(\mathrm{H}_{0}\right)$ proposes there will be no significant influence of physical activity participation and lifestyle choice on body image perception and awareness.

\section{Introduction}

The behaviour and attitude of an individual towards themselves, both in independent and social scenarios, vary widely across different personalities and personas. The level of physical activity (PA) participation and types of lifestyle choice are both shown to have a significant influence on one's psychological wellbeing and self-perception [1,2]. When exposed to a social situation, external judgements of body image and physical presentation, of which heightens social physical anxiety (SPA) and increased body awareness of one's imperfections. This can lead to decreased self-confidence, motivation, and reduced self-esteem [3]. However, taking part in exercise has been found to reduce the subconscious concern towards self-assessing physical appearance, to avoid the potential judgements and accusations made by other people [4].

Self-presentation involves the inclusion and exclusion of specific aspects, personally and environmentally, to create the bestdesired impression of oneself and avoid undesired perceptions [3]. External/surrounding factors, which may involve a social encounter inhibiting one's confidence in their physical appearance; of which has been heavily associated with exercise participation and the interpersonal context [5]. Through assessing the relationship between psychological awareness of one's body image in internal, individual and social conditions, and varying PA levels the degree of significance can be extrapolated. Leary and Kowalski found sedentary people withhold lower self-confidence and self-esteem due to a lack of motivation and self-drive, as the negative impression of themselves has developed over time, resulting in a poor bodily self-perception [3].

Body dissatisfaction is the negative impression one builds in response to their body image and the low subjective evaluation of the weight and shape of their body. This is experienced by sedentary and low exercisers, leading to social involvement issues, isolation, and confidence barriers [6]. PA has been shown to heighten confidence [7], construct positive identities and improve quality of life [8]. A comparison between sedentary people, the intermediate exercise groups (socially and casually) and competitively active people will provide suitable depth and reliability, covering the main PA levels, relative to lifestyle and personal motivation.

\section{Methods \\ Participants}

The target population was healthy students aged 18 - 21 of any sporting/physical activity backgrounds, male or female, from the University of Hertfordshire. The age restriction was the main inclusion criteria, as this is the progression of late adolescence into early adulthood and is a pivotal time, filled with uncertainty, for body image awareness [9]. The exclusion criteria for the study involved; participants who played a sport nationally could not be used, as in 
a more elite environment, psychological coaches can assist athletes to overcome any mental issues, which may improve or hinder one's body image perception. No participant could have experienced an injury in the last 6 months before data collection, as this could have altered self-presentation and confidence in their [10]. No participant could suffer from a psychological or physiological illness as this would have inhibited one's view and opinion of themselves.

The study was reviewed and approved by the University of Hertfordshire Health and Human Sciences Ethics Committee with Delegated Authority. The UH protocol number of the study was cLMS/GP2/UH/05001. The Risk Assessment (HRSA) covered the potential risks involved with the activities involved in the study and was signed off by my Supervisor and the Laboratory Health and Safety Manager. The letter of confirmation was provided to approve subject recruitment and data collection taking place within the Learning Resource Centre (LRC).

All participants involved in the study initially volunteered their services when recruitment took place in the LRC foyer, of which was random opportunistic sampling, volunteering to be a part of the investigation, using an enticing table advertisement, over a 6-week period. Upon this first meeting, students were given a Participant Recruitment (PR) Form, which asked questions concerning demographic information and one's average physical activity levels during a typical week, of which was used to dictate test group (Sedentary; Social Active; Casual Active; Competitive Active) [11]. The participants who met the inclusion criteria for the study were contacted up to four weeks later regarding data collection. Upon meeting the respective participants, a Participant Information Sheet (EC6) was provided to explain the study and their involvement with the research project, at which point, one had the option to leave if they wished. Following this, the participants gave their written informed consent through the ethical Consent Form (EC3), agreeing to the terms and conditions of the study, volunteering their involvement.

\section{Procedural design}

The study involved quantitative data collection through the means of three published questionnaires, through a descriptive cross-sectional design, collecting data from participants on a single occasion. Prior to the main data collection, a pilot test was carried out on several peers to judge the effectiveness and influence of the questionnaires; everyone responded with positive remarks, stating how they gave them a chance to self-assess and self-reflect [12].

The LRC study rooms were booked out on several occasions to provide the opportunity to the participant, upon meeting the inclusion criteria, of dropping by and filling the consent forms and questionnaires, which occurred over an 8-week period. If a participant was unavailable or busy, the questionnaires would be sent to them via email. Necessary questionnaire results or feedback were supplied to a participant if they requested so, to help their own perceptions and self-discovery of body image [1].

The measurement equipment involved three published questionnaires (Likert Scales). The Body Awareness Questionnaire (BAQ), which measured the beliefs of one's sensitivity and type of response in a personal sense, for specific scenarios, and their own intrinsic reaction to an experience or situation (13). The Body Consciousness Questionnaire (BCQ), which measured the private and public body consciousness (internal sensations), looking at the observable aspects of the body, relative to the effectiveness of physical movements [10]. The Social Physique Anxiety Scale (SPAS) assessed the degree to which one becomes anxious when others observe or evaluate body image, by measuring their characteristic response in various social scenarios [2].

In recruitment, upon requesting for one's typical weekly activity levels on the PR form, no mention of the test groups was relayed to them, as this may have altered their honesty in responding to the requirement. The participant sampling method was opportunistic random, attempting to recruit anyone who passed the table in the foyer, who was within the age inclusion criteria. All questionnaire authors provided the validity and reliability of their respective work.

\section{Data analysis}

Quantitative data was collected and stored anonymously in a locked cabinet and a password-protected laptop. Using G*Power software [14], a power test was undertaken to dictate the estimated sample size, in relation to the statistical analysis, a one-way fixed-effects ANOVA (analysis of variance). The effect size was 0.40 (large effect), the alpha value was set at 0.05 and power at 0.80 [15], relative to the four test groups. Using IBM SPSS Inc. for Windows, version 17.0 (released in 2008), the parametric data (Likert 
scales produce ratio and interval results) for all questionnaires was assessed by Shapiro-Wilk Test of Normality ( $p>0.05)$. A one-way fixed-effect ANOVA statistical test was used to compare the differences between the four test groups of the varying physical activity participation levels, for each individual questionnaire [16], and concerning each hypothesis.

\section{Results}

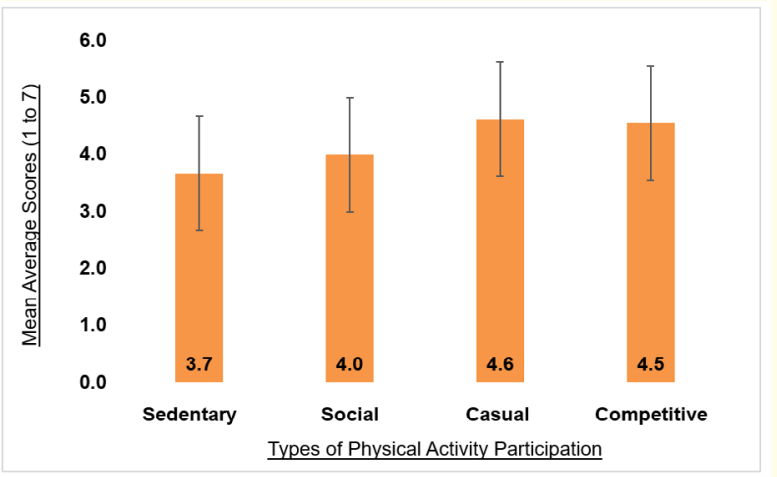

Figure 1: A comparison between the mean average scores on the body awareness questionnaire (BAQ) for all participants in relation to each extent of physical activity participation on a weekly basis, including standard deviations. The orange bars on the average scores, with the vertical lines showing the range of scores from participants.

Figure 1 showed a lower internal concern for physiological functions and reactions to both their subconscious realisation of bodily changes and in certain scenarios with the lowest raw average for a participant being 2.2 out of 7. The Casual and Competitive Active groups both required a higher apprehensive approach to physical activity, as going to the gym or exercising personally (Casual) involved elevated self-motivation and when competing for a sports (University) team; the drive is naturally raised to reach the achievement outcome. This was shown through the two highest individual average scores being 5.5 and 5.9 out of 7 in the Competitive and Casual groups, respectively. The Social Active group showed a moderate score, as they are playing for the enjoyment factor and may show a slight consideration of their body's intrinsic responses and reactions.

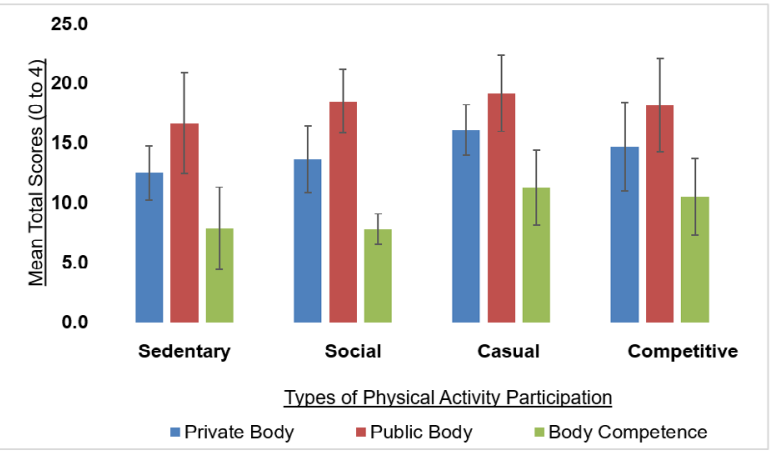

Figure 2: A comparison between the mean total scores on the body consciousness questionnaire (BCQ) for all participants, in terms of the three subscales, in relation to each extent of physical activity participation on a weekly basis, including standard deviations.

Figure 2 demonstrated a progression in Private Body consciousness (internal bodily sensations) and then a drop off for the Competitive group, which is similar to the BAQ results in figure 1 . The Competitive group had the largest standard deviation (of \pm 3.7 ), which may be relative to training age in the activity or, individual compared with team sports. Public Body consciousness was a constant through all test groups, as it entails bodily appearance as perceived and judged by an observer, which is reflective of the age inclusion criteria for the study (18 - 21 years). The Sedentary group was the lowest (at an average of 16.7 out of 24), as the routine of little to no physical activity has become a habit and psychological adjustment, creating a negative impression of themselves. The Social group had the highest individual score of 24 out of 24, which is indicative of their lower ability and peer pressure in a non-competitive activity. Body Competence is an individual's sense of effectiveness in movements or as a person, which naturally corresponds with physical activity, hence the higher average scores for Casual. The Competitive group may already be aware of their strengths and weaknesses, having played at a higher level, showing reduced consciousness awareness of movement efficiency. This tied in with the lowest individual score in the Sedentary group being 2 out of 16 for Body Competence. 


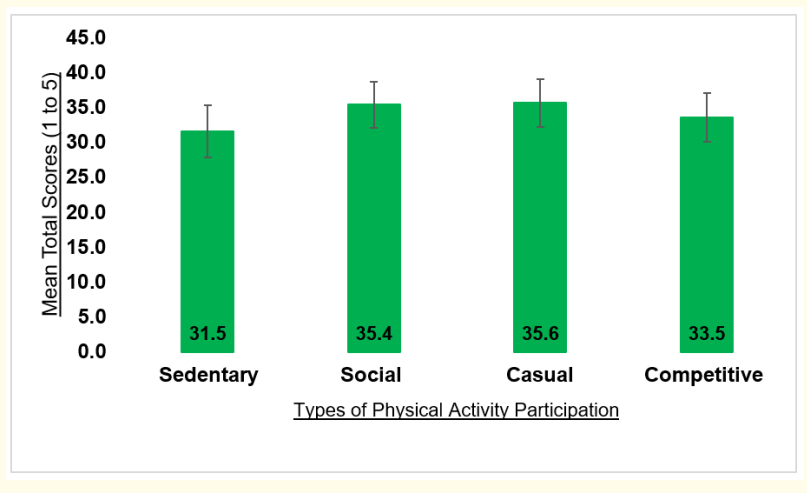

Figure 3: A comparison between the mean total scores on the social physique anxiety scale (SPAS) for all participants, in relation to each extent of physical activity participation on a weekly basis, including Standard Deviations.

Figure 3 presented the increased self-confidence in body image from a Competitive participant, as they may maintain a suitable physique for sporting performance. The Social and Casual participants are playing with friends or for personal goals, not necessarily for performance outcomes, showing a presence of anxiety in one's physical appearance in social scenarios, as they may wish to one day compete at a higher level. The highest individual score was in the Social Active group, 42 out of 48. The SPAS displayed how social physique anxiety was ever-present throughout the student body, as Sedentary people may be insecure about their appearance and Competitive performers may be struggling to sustain or maintain body composition in order to excel in their desired physical activity/sport.

Relative to the one-way ANOVA, a power test was undertaken using $\mathrm{G}^{*}$ Power software to produce a sample size of $n=76$, to achieve significance (large effect size), showing the study as underpowered $(n=28)$. The Shapiro-Wilk test confirmed the parametric data set as normal for each questionnaire, relative to the four test groups ( $\mathrm{p}>0.05$ ). The one-way ANOVA for each questionnaire compared the four independent variables (physical activity participation) and produced no statistically significant differences between the means. The BAQ produced $[\mathrm{F}(3,24)=2.52$, $\mathrm{p}=0.082]$; the BCQ produced $[\mathrm{F}(3,24)=2.680, \mathrm{p}=0.070]$ and the SPAS produced $[\mathrm{F}(3$, $24)=2.049, p=0.134]$ respectively. Concerning the hypotheses (page 13) one-way ANOVAs, hypotheses 1 and 4 were not statically significant. Hypothesis 2 (Social vs. Casual Active on the BCQ) was statistically significant; $[\mathrm{F}(1,14)=4.68, \mathrm{p}=0.048]$. Hypothesis 3 (Sedentary vs. Casual for BCQ) was also statistically significant; $[F(1,12)=6.68, p=0.024]$.

\section{Discussion}

Body image refers to both the psychological picture in one's mind of their perceived physical appearance and the external factors, influences and reasons for the poor body confidence, negative feelings, and emotion [17]. The level of physical activity (PA) participation [11] and types of lifestyle choice are shown to have a significant influence on one's psychological wellbeing and body image awareness $[1,2]$. The aim of this study was to investigate the extent of a relationship between the level of physical exercise undertaken during a typical week and one's lifestyle choice on body image perception and awareness in University students aged 18 - 21. The psychological response to physical reactions and bodily tensions [10] and attitudinal behaviours towards one's body is significantly influenced by PA level [18] in both individual and social scenarios.

Multiple studies used the questionnaire approach as an intervention to a psychological state or perception, to create comparative data (before and after) at two different time points [16,19]. There is research looking into age range and body image but not specifically, both in terms of solely body image and for a succinct range of 18 - 21 years, with studies investigating participants aged 18 - 25 [20] and adolescent individuals aged 18 - 21 [9]. This was shown in figure 2 to be a heavily associated age range with body consciousness, as all PA levels produced moderately high scores in the 'Public Body' sub-scale, implying self-presentation to others is for social acceptance, as they are most susceptible to the media [12]. Although there was no statistical significance between the four test groups within each questionnaire, meaning the null hypothesis has been accepted, although there were two significant hypotheses.

The inclusion height and weight measurement for differing physiological backgrounds [21] were considered for this methodology as direct trends and relationships relative to declining activity levels were found. Although PA is shown to have a significant psychological impact, with a sense of achievement and self-reward from a good performance or reaching a new personal best [22], to improve confidence in oneself; to improve the overall quality of life [8]. 
PA is undertaken by various people for social inclusion and to feel a sense of community and friendship [6,23], which was reflective of the study, as the most populated test groups were the Social and Casual active participants, playing for enjoyment and personal achievement. Hypothesis 2 was statistically significant, relative to the $\mathrm{BCQ}[\mathrm{F}(1,14)=4.68, \mathrm{p}=0.048]$; presenting how in relaxed and non-competitive, the main focus is the social aspect, not an achievement outcome or long-term goal. The BAQ, BCQ and SPAS have had positive effects on body image and social physique anxiety in studies based around PA, but through a limited PA type spectrum $[16,24]$.

The intrinsic response, relative to body appearance, is viewed to be a consequence of PA participation [2], resulting in positive effects on self-esteem and encouraging the rejection of external judgement. This was shown in figure 1, with the socially and casually active groups showing heighten body image assessment and mindfulness, whereby several participants were simply proactive and attended a society or scheme. This was the focus of the SPAS, encouraging self-assessment of self-presentation and the potential insecurities relative to external judgement $[4,25]$. The BCQ ties this 'public body' perception in with a 'private body' and 'body competence' sub-scales, to show the differing adaptive behaviours [5]; which may lead to fear or uncertainty for sedentary individuals, due to low self-confidence [26]. This negative impression development has evolved through past experiences and the number of opportunities on offer previously, of which were not considered in this study, although is evident in figure 1 and 3 , with the sedentary group being surprisingly low. Hypothesis 3 supported this trend, as statistically significant, $[\mathrm{F}(1,12)=6.68, \mathrm{p}=0.024]$; there is reduced body image awareness in both a private and public sense for sedentary individuals. This is due to psychological adjustment, with the inactive lifestyle becoming a habit and the motivation levels remain low [3], sourcing easy achievement from simply, less active tasks; this may have mental implications and build a fearful resistance to involvement. Sedentary adolescents may also have yet to exercise in their leisure and therefore not appropriately informed about the negative effects of not exercising [27]. In the long term, sedentary and low exercisers can develop social involvement issues, isolation and confidence barriers [6].

The Social and Casual groups presented the highest mean total scores for the SPAS, as the motivation would be to play against friends or to reach performance goals on a personal note, increas- ing the inclination to external judgement by peers and having an added pressure in social scenarios to present a positive and successful body image [17]. Whereas Competitive individuals may have reached a suitable physique for their sport and will not be afraid to take part within social environments and judgemental surroundings [18], with a lower SPAS score, although there remains pressure from coaches and managers, as the achievement outcome may be the focus [2]. In terms of all questionnaires used $[2,10,13]$, there seems to be a moderately strong relationship between PA participation and lifestyle choice, relative to body image awareness.

The westernised views of diet, body composition and the ideal body, which at the ages of 18 - 21, the opposite sex and physical presentation become the forefront of daily life. The media and culture present unrealistic body shapes, of which deflates self-esteem and self-confidence [12]. The lifestyle and personal freedom, particularly in students, was a significant factor; they may be away from home for the first time and have many different opportunities and lifestyle choices on offer. Research has shown from the ages of 13 - 21, there is a gradual decline in PA participation [9]. Relative to social inclusion, the opposite sex may desire a particular body type as a result of the media [20] creating further barriers and difficulties for an inactive, unfit individual to become involved with PA, the public eye's physical acceptance [28].

More research into body image relative to age range is required [29], as the media is reaching children at a younger age and promoting the 'perfect' body to aspire to, which can have consequential impacts psychologically [30]. The comparison of gender in relation to PA is a growing area and has presented connections with poor body perception and low motivation levels [4,31] and the level of competition. Research has shown competitive athletes to show heightened competence and self-discipline but also had tendencies for appearance-related motives [32]. In terms of testing measures, self-esteem has been a common reference in multiple studies; testing this using the Rosenberg Self-Esteem Scale [33], which would relate the PA to more than just body image. Through grouping participants relative to their PA level, a further consideration to make in future research would be why have they chosen to compete/play at this level? What are their motivations? Using the Exercise Motivations Inventory (EMI-2) [34], which addresses external purposes for PA, such as social recognition, enjoyment, and stress/weight management.

Citation: Lewis Bridges and Dr Stephen Pack. "To Investigate the Extent of the Level of Physical Activity Participation and Lifestyle Choice having a Significant Influence on Body Image Perception and Awareness in University Students Aged 18 - 21". Acta Scientific Orthopaedics 4.6 (2021): $46-54$. 
The limitations of the study involved a lack of depth in connecting with the participants on a psychological level, as questionnaires discover information relative to the theme and question areas (Likert Scales); interpersonal investigations may be more detailed through qualitative research. This may involve the participant's PA history (training age) and their diet/nutritional intake, which is just as significant as the physiological side. There were small participant numbers and a poor response rate - opening up the study to more than students may allow recruitment to grow, allowing the potential to investigate gender, as the test groups were unbalanced in males and females. In terms of measurement, the questionnaires were slightly dated, and research has developed and widened since the 1980s; using a newer scale or inventory may be more beneficial [19].

At a Masters or PhD level, this study could be broadened to accommodate more participants, more questionnaires/scales of assessment and therefore, increased dependent/test variables, to include psychological components such as self-esteem, confidence, and wellbeing. This study could be taken further to investigate or eliminate external factors, such as investigating physical/psychological backgrounds, medical history, and more in-depth lifestyle involvement, such as whether one commutes or drives. Similarly, the type of sport, individual or team, has shown relationships and differences in body consciousness and attitudes towards selfpresentation [35]. The effect of injury on body image, potentially pre-and-post the injury event for a competing athlete could have significant implications and detrimental wellbeing inhibitions for future rehabilitation.

\section{Conclusion}

The level of physical activity participation and lifestyle choice does have a moderate influence on body image perception and awareness in independent and social scenarios, as shown by the BAQ and BCQ. The SPAS presented a smaller inclination of appearance anxiety in social environments; this may be as a result of the inactive lifestyle and psychological adjustment.

The vulnerability of sedentary people to shy away from PA, due to the fear of social scenarios and being physically judged through adaptive behaviours, leads to a sense of comfort yet avoidance, encouraging sedentary tasks to be undertaken [5]. This low body self-confidence [26] is related to social physique anxiety and body awareness, creating a negative impression of themselves [3], which is assessed through the BAQ, BCQ and SPAS. Numerous studies do not compare the sedentary individuals with the physically active [21,31]; hence the four test groups. The inclusion criterion of being 18 - 21 years old is significant; when they are most susceptible to the media $[12,30]$ and surrounding body types, with many studies assessing middle-aged participants [20] and teenagers [9].

Further research into body image relative to PA participation is required, to eliminate inhibiting factors such as diet, occupation, and location of residence (particularly for students), as participants who commuted tended to have reduced involvement in University sports clubs and societies. Assessing further psychological cognitions, such as self-esteem and exercise motivations would be beneficial to body image research.

\section{Acknowledgements}

I would like to sincerely thank my project supervisor, Dr Stephen Pack, for the help, support, and advice he has generously provided during this project, both in supervisor meetings and over email, acting as a guide and mentor. Thank you to Sally Maurice for providing permission to carry out participant recruitment and data collection for this research project in the College Lane Learning Resource Centre study rooms and foyer at the University of Hertfordshire. I would also like to thank Scott Grace for processing and arranging the use of the Forum Hertfordshire for participant recruitment. A further thank you extends to Mr Joel Harris, as Module Leader, for overseeing the cohort's project process and efficiently replying to any queries or concerns raised by myself and fellow students. Finally, thanks to my family and friends who have wholeheartedly supported my efforts during the four years of this degree course, particularly during my final year.

The study was reviewed and approved by the University of Hertfordshire Health and Human Sciences Ethics Committee with Delegated Authority. The UH protocol number of the study was cLMS/GP2/UH/05001.

\section{Bibliography}

1. Bartels M., et al. "Regular exercise, subjective wellbeing, and internalizing problems in adolescence: causality or genetic pleiotropy?". Frontiers in Genetics 3 (2012): 4.

2. Hart EA., et al. "Tie Measurement of Social Physique Anxiety". Journal of Sport and exercise Psychology 11.1 (1989): 94-104. 
3. Leary MR and Kowalski RM. "Impression management: A literature review and two component model". Psychological Bulletin 107.1 (1990): 34-47.

4. Bain LL., et al. "Participant perceptions of exercise programs for over weight women". Research Quarterly for Exercise and Sport 60.2 (1989): 134-143.

5. Leary MR. "Self-presentational processes in exercise and sport". Journal of Sport and Exercise Psychology 14.4 (1992): 339-351.

6. Ellis N., et al. "Exercise interventions as an adjunct therapy for psychosis: a critical review". British Journal of Clinical Psychology 46.1 (2007): 95-111.

7. Hodgson MH., et al. "The experiences of people with severe and enduring mental illness engaged in a physical activity programme integrated into the mental health service". Mental Health and Physical Activity 4.1 (2011): 23-29.

8. Acil AA., et al. "The effects of physical exercises to mental state and quality of life in patients with schizophrenia". Journal of Psychiatric and Mental Health Nursing 15.10 (2008): 808-815.

9. Telama RISTO and Yang X. "Decline of physical activity from youth to young adulthood in Finland". Medicine and Science in Sports and Exercise 32.9 (2000): 1617-1622.

10. Miller LC., et al. "Consciousness of body: Private and public". Journal of Personality and Social Psychology 41.2 (1981): 397406.

11. Abbott BD and Barber BL. "Differences in functional and aesthetic body image between sedentary girls and girls involved in sports and physical activity: Does sport type make a difference?" Psychology of Sport and Exercise 12.3 (2011): 333-342.

12. Grabe S., et al. "The role of the media in body image concerns among women: a metaanalysis of experimental and correlational studies". Psychological Bulletin 134.3 (2008): 460-476.

13. Shields SA., et al. "The body awareness questionnaire: reliability and validity". Journal of Personality Assessment 53.4 (1989): 802-815.
14. Faul F., et al. "Statistical power analyses using G* Power 3.1:Tests for correlation and regression analyses". Behavior Research Methods 41.4 (2009): 1149-1160.

15. Cunningham JB and McCrum-Gardner E. "Power, effect and sample size using GPower: practical issues for researchers and members of research ethics committees". Evidence-Based Midwifery 5.4 (2007): 132-137.

16. Daubenmier JJ. "The relationship of yoga, body awareness, and body responsiveness to self-objectification and disordered eating". Psychology of Women Quarterly 29.2 (2005): 207-219.

17. Brodie DA., et al. "Body-image perception in pre-and postadolescent females". Perceptual and Motor Skills 78.1 (1994): 147-154.

18. Skrinar GS., et al. "Effects of endurance training on body consciousness in women". Perceptual and Motor Skills 62.2(1986):483-490.

19. Knauss C., et al. "Body dissatisfaction in adolescent boys and girls: Objectified body consciousness, internalization of the media body ideal and perceived pressure from media". Sex Roles 59.9-10 (2008): 633-643.

20. Demarest J and Allen R. "Body image: Gender, ethnic, and age differences". The Journal of Socialpsychology 140.4 (2000): 465-472.

21. Loland NW. "Body image and physical activity. A survey among Norwegian men and women". International Journal of Sport Psychology 29.4 (1998): 339-365.

22. Schlenker BR and Leary MR. "Social anxiety and self-presentation: A conceptualization model". Psychological Bulletin 92.3 (1982): 641-669.

23. Gidlow C., et al. "Attendance of exercise referral schemes in the UK: a systematic review". Health Education Journal 64.2 (2005): 168-186.

24. Rani NJ and Rao PK. "Body awareness and yoga training". Perceptual and Motorskills 79.3(1994):1103-1106. 
25. Siegel PZ., et al. "The epidemiology of walking for exercise: implications for promoting activity among sedentary groups". American Journal of Public Health 5.5 (1995): 706-710.

26. Crawford S and Eklund RC. "Social physique anxiety, reasons for exercise, and attitudes toward exercise settings". Journal of Sport and Exercise Psychology 16.1 (1994): 70-82.

27. Nigg CR and Courneya KS. "Transtheoretical model: Examining adolescent exercise behavior". Journal of Adolescent Health 22.3 (1998): 214-224.

28. Pope HG., et al. "Evolving ideals of male body image as seen through action toys". International Journal of Eating Disorders 26.1 (1999): 65-72.

29. Demarest J and Langer E. "Body shape satisfaction: Sex and age differences across the lifespan". Manuscript Submitted for Publication (1997).

30. Agliata D and Tantleff-Dunn S. "The impact of media exposure on males' body image". Journal of Social and Clinical Psychology 23.1 (2004): 7-22.

31. McDermott L. "A qualitative assessment of the significance of body perception to women's physical activity experiences: Revisiting discussions of physicalities". Sociology of Sport Journal 17.4 (2000): 331-363.

32. Nia ME and Besharat MA. "Comparison of athletes' personality characteristics in individual and team sports". Procedia-Social and Behavioral Sciences 5 (2010): 808-812.

33. Rosenberg M. "Society and the Adolescent Self-Image. Revised. Middletown, CT: Wesleyan University Press (1989).

34. Ingledew DK., et al. "Exercise motives and stages of change". Journal of Health Psychology 3.4 (1998): 477-489.

35. Molanorouzi K., et al. "Motives for adult participation in physical activity: type of activity, age, and gender". BMC Public

Health 15.1 (2015): 66.

\section{Volume 4 Issue 6 June 2021}

C) All rights are reserved by Lewis Bridges and Dr Stephen Pack. 